\title{
Study of the magnetic turbulence in a corotating interaction region in the interplanetary medium
}

\author{
J. F. Valdés-Galicia, R. A. Caballero \\ Instituto de Geofísica, UNAM, 04510 México, D.F., México \\ E-mail: jfvaldes@tonatiuh.igeofcu.unam.mx \\ Received: 16 July 1998 / Revised: 9 June 1999 / Accepted: 5 July 1999
}

\begin{abstract}
We study the geometry of magnetic fluctuations in a CIR observed by Pioneer 10 at 5 AU between days 292 and 295 in 1973 . We apply the methodology proposed by Bieber et al. to make a comparison of the relative importance of two geometric arrays of vector propagation of the magnetic field fluctuations: slab and two-dimensional (2D). We found that inside the studied CIR this model is not applicable due to the restrictions imposed on it. Our results are consistent with Alfvenic fluctuations propagating close to the radial direction, confirming Mavromichalaki et al.'s findings. A mixture of isotropic and magnetoacoustic waves in the region before the front shock would be consistent with our results, and a mixture of slab/2D and magnetoacoustic waves in a region after the reverse shock. We base the latter conclusions on the theoretical analysis made by Kunstmann. We discuss the reasons why the composite model can not be applied in the CIR studied although the fluctuations inside it are two dimensional.
\end{abstract}

Key words. Solar physics, astrophysics and astronomy (magnetic fields) - Space plasma physics (turbulence; waves and instabilities)

\section{Introduction}

Discussion of the geometry of the interplanetary magnetic field (IMF) fluctuations usually refers to the slab model because we think in terms of Alfvèn waves propagating along the mean magnetic field. In this model, fluctuation wave vectors are aligned with that field. However, in recent years some published work provides for the presence of the fluctuation of IMF with a two dimensional (2D) component (Matthaeus et al., 1990, 1995). In the case of 2D turbulence the wave

Correspondence to: J. F. Valdés-Galicia vectors are aligned preferentially in the plane perpendicular to the mean magnetic field direction. Consequently, a composite model was proposed, by Matthaeus et al. (1990) and Bieber et al. (1996) that included slab and 2D components, to explain the observed fluctuations in the IMF.

Laboratory plasma experiments motivated studies of possible $2 \mathrm{D}$ turbulence in the interplanetary medium. In particular, Robinson and Rusbridge (1971) studied the fluctuations of electric and magnetic fields in the Z-pinch instability, and found that correlation scales along the mean field were much longer than those perpendicular to the mean field. This very large anisotropy in the turbulence resembled bidimensional turbulence in fluids according to these authors. Experiments in tokamaks provided another test of bidimensional turbulence: Zweben et al. (1979) found that the correlation length in small-scale fluctuations of the magnetic field along the toroidal direction is considerably larger than the length in poloidal and radial directions.

Moreover, thorough MHD numeric simulations by Oughton et al. (1994) showed an asymmetry in the fluctuations, with the main transfer of energy to wave vectors that are transversal with respect to the mean field. This is a proof that 2D fluctuations exist, when turbulence is fully developed.

In the studies of cosmic ray transport the slab model has figured predominantly (Jokippi, 1966; Hasselmann and Wibberenz, 1968). As is known, there is a longstanding discrepancy between the mean free path derived from the theories and the calculated value based on solar proton event profiles (Kunow et al., 1991; Valdés-Galicia, 1993). Recent studies (Bieber et al., 1994, 1996) have shown that this old problem may be resolved if a turbulence composite slab/2D model with dominant $2 \mathrm{D}$ component $(\sim 80 \%)$ is able to fit the observations. This is another motivation to investigate the geometry of the magnetic fluctuations and especially the bidimensional model.

We study the geometry of magnetic fluctuations in a CIR observed by Pioneer 10 at 5 AU between days 292 
and 295 in 1973 . We apply the methodology proposed by Bieber et al. (1996) allowing a comparison of the relative importance of two geometric arrays of vector propagation of the magnetic field fluctuations: slab and 2D. In Sect. 2 the data and the techniques selected to analyse them are presented. In Sect. 3 we give a brief explanation of the turbulence model, and how we applied it to our data. Section 4 contains the results of the work. There is a discussion in Sect. 5, and our conclusions are given in Sect. 6 .

\section{Data selection}

The data used are magnetometer measurements taken on board Pioneer 10 spacecraft at 5 AU from the Sun. The period of observation corresponds to the days between 287 and 300 in 1973. In Fig. 1 we show hourly averages of the IMF for this time period.

Some of the original data we received (by courtesy of Prof. E. Smith, JPL, NASA) have a time resolution of up to $0.375 \mathrm{~s}$ and contain the three components of the IMF in an RTN system of coordinates (the $x$ component in the radial direction, the $y$ component in the transversal direction in the ecliptic plane and the $z$ component in the normal direction). However, as a major part of the data had a temporal resolution of $0.75 \mathrm{~s}$, we selected that resolution for our work. Periods of data with smaller time resolution were accordingly decimated. This allowed our analysis to be completely in the inertial range, since the cutoff frequency for this time resolution is very close to the limit boundary with the dissipative range $(\sim 1 \mathrm{~Hz})$. The precision within each IMF component is $0.001 \gamma$.

Each array contained 1024 data giving enough statistical reliability, (this corresponds to $768 \mathrm{~s}$ or $13 \mathrm{~min}$ ). In this manner we were able to complete 445 sets of data with a temporal resolution of $0.75 \mathrm{~s}$ where the data gaps were not greater than $10 \mathrm{~s}$. Gaps were linearly interpolated. The usual spectral techniques require the data to represent an ergodic and stationary process in order to have reliable power spectral estimates. The criterion that the autocorrelation function must be of the Lanczos type guarantees that the power spectra obtained are reliable (Matthaeus and Goldstein, $1982 \mathrm{a}, \mathrm{b})$. Therefore, we verified that for a lag between $40 \mathrm{~s}$ and $80 \mathrm{~s}$, approximately, the autocorrelation function decreases to $10 \%$ of its value for zero lag. We calculated the autocorrelation function for each component of the 445 arrays and analysed the behaviour of the trace of $R_{i j}(L)$, where $L$ is the lag, using the Blackman-Tukey technique to calculate the autocorrelation (Blackman and Tukey, 1958). A stationary behaviour was observed in only 284 arrays, these constituted our work set. The stationary arrays were divided into three intervals for its analysis: (1) the period before the forward shock, known as "before the CIR", with 118 arrays; (2) the period of time between the forward and reverse shocks, or "inside the CIR", with 68 arrays; and (3) the period after the reverse shock crossed the spacecraft, referred to as "after the CIR" with 98 arrays. The convergence test (see Sect. 3) was also made for the samples "before" and "after" the CIR. The division was done with the aim to analyse separately

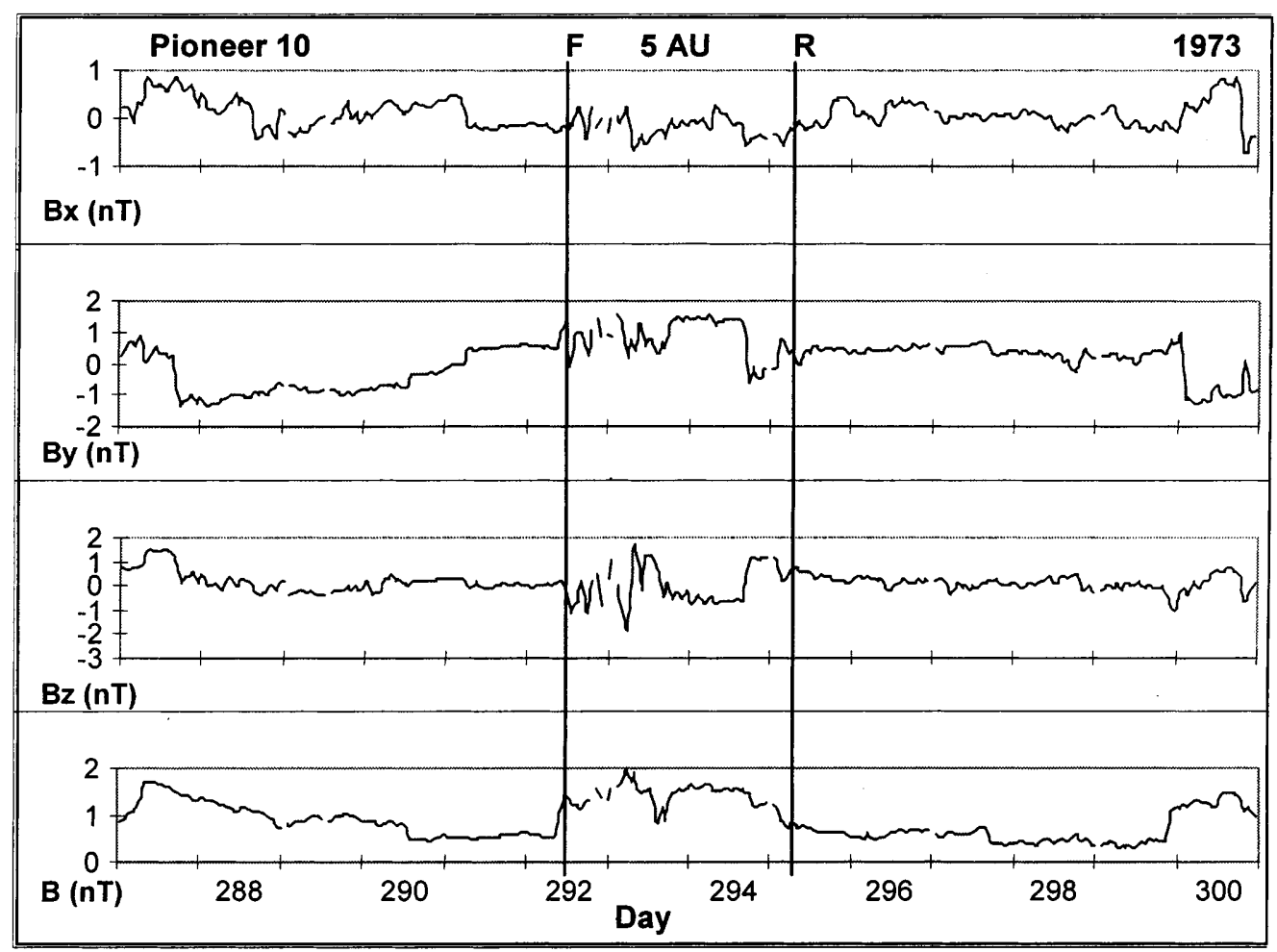

Fig. 1. Cartesian components and magnitude of the IMF measured by the Pioneer 10 at 5 UA. We present hourly averages. The forward shock of the CIR $(F)$ appears about 11:15 on day 292, while the reverse shock $(R)$ is around 03:20 on day 295 
the behaviour of the magnetic fluctuations before, during and after the CIR and detect possible differences since they constitute three separate magnetic regimes.

\section{Application of the composite slab/2D model}

The composite slab/2D model assumes that:

1. The magnetic fluctuations are alfvenic.

2. The magnetic turbulent field $\vec{B}(t, \vec{x})$ may be decomposed as a sum of the mean magnetic field $\vec{B}_{0}$ and fluctuating field $\vec{b}$ with mean value equal to zero.

3 . The turbulence is homogeneous. This implies that the correlation function does not depend from spatial position, that is:

$R_{i j}(\vec{r})=\left\langle b_{i}(\vec{x}) b_{j}(\vec{x}+\vec{r})\right\rangle$

is independent of the choice of $\vec{x}$. The power spectrum:

$P_{i j}(\vec{k})=\frac{1}{(2 \pi)^{3}} \int d^{3} r R_{i j}(\vec{r}) e^{-i \vec{k} \vec{r}}$

only depends on $\vec{k}$.

4. The turbulence is axisymmetric with respect to the mean magnetic field. We can describe the spectral decomposition in terms of the parallel component of the wave vector, $k_{\|} \equiv \hat{z} \cdot \vec{k}$, and the perpendicular component $k_{\perp} \equiv|\underline{\hat{z}} \times \underline{\vec{k}}|$. In the slab case the spectra only depend on $k_{\|}$, while $k_{\perp} \approx 0$. In the $2 \mathrm{D}$ model the spectra depend on $k_{\perp}$, and $k_{\|} \approx 0$. Here the axis $z$ is along the direction of $\vec{B}_{0}$. The condition of axisymmetry implies that the tensor $P_{i j}(\vec{k})$ is invariant with respect to the rotation about the direction of the mean magnetic field, i.e., about the $z$ axis.

5. The power of the magnetic fluctuations along the direction of the mean magnetic field is smaller than the power of the fluctuations in the plane perpendicular to this direction.

Bieber et al. (1996) analysed the functional form of the power spectra for each of the geometries, slab and 2D. They denote $P_{\|}^{\prime}=P_{x x}$ to the radial direction spectrum that they call "quasi-parallel" because it has a component along the sampling direction, and $P_{\perp}=P_{y y}$, the spectrum perpendicular to the radial direction. By making a ratio of the two components of the composite model one obtains:

$P_{\perp} / P_{\|}^{\prime}=\frac{(\cos \psi)^{q-1}+\left(\frac{1-r}{r}\right)\left(\frac{2 q}{1+q}\right)(\sin \psi)^{q-1}}{(\cos \psi)^{q-1}+\left(\frac{1-r}{r}\right)\left(\frac{2}{1+q}\right)(\sin \psi)^{q-1}}$

where $r=C_{S} /\left(C_{S}+C_{2 D}\right)$ is a constant ratio of amplitude components.

If we know the angle between the radial direction and the direction of the mean magnetic field $(\psi)$, we may calculate the value of $r$ for the best fit of the theoretical model to the observations. In this manner we may compute the proportion of each type of fluctuation in the IMF. If we have only slab type fluctuations, $r=1$, and the the ratio of spectra will be 1 . If the fluctuations are purely $2 \mathrm{D}, r=0$, and the ratio of the spectra will be equal to the spectral index $q$. Hence, in the inertial range, Kolmogoroff (1941) theory predicts $r=-5 / 3$.

Bieber et al. (1996) applied the model to IMF data from the Helios spacecrafts at heliocentric distances between 0.3 and 1 AU. For this analysis they used 454 arrays of 34 min each with a temporal resolution of $8 \mathrm{~s}$. The frequency range analysed was between $9.8 \times 10^{-4} \mathrm{~Hz}$ and $2.05 \times 10^{-2} \mathrm{~Hz}$. They calculated the ratio $P_{\perp} / P_{\|}^{\prime}$ for each spectrum and obtained the geometric mean value of this ratio as 1.4. The ratio is larger than unity (slab model) and closer to 1.67 as predicted by the 2D model. This result is an evidence of an important presence of bidimensional fluctuations.

Since one of the assumptions of the model is that the turbulence is axisymmetric, we calculated power spectra in a system of reference that is rotated in relation with the RTN system. The new system of rectangular coordinates $\left(x^{\prime}, y^{\prime}, z^{\prime}\right)$, is oriented in the following manner: the $x^{\prime}$ axis is in the direction of the average magnetic field and pointing away from the Sun; the $z^{\prime}$ axis is in the plane that contains the mean magnetic field and the radial direction, that is, contains the axes $x^{\prime}$ and $x$, and also points away from the Sun; and the $y^{\prime}$ axis completes the right handed system in such a manner that it is perpendicular to the plane that contains the direction of the mean magnetic field and the radial direction. We denote $\left(b_{x m}, b_{y m}, b_{z m}\right)$ the components of the mean magnetic field with magnitude $b_{m}$ in the RTN system, and $\left(b_{x}^{\prime}, b_{y}^{\prime}, b_{z}^{\prime}\right)$ the components of the field in the new system.

In our case, the direction of the mean magnetic field is the direction of the $x^{\prime}$ axis, $\vec{b}_{m}^{\prime}=\left(b_{x m}, 0,0\right)$. The direction $y^{\prime}$ is the direction of the perpendicular fluctuations $\left(P_{\perp}\right)$, while the direction $z^{\prime}$ is the direction of the fluctuations quasi-parallel to the sampling direction of $P^{\prime}$.

We calculated the values of the mean IMF orientation for each of the 284 arrays, with respect to the radial direction $(\psi)$ to complete the transformation of coordinate systems. After the passage of the forward shock, the mean magnetic field rotates towards large angle directions with respect to the radial direction, in contrast with the data used by Bieber et al. (1996) where the values of the mean magnetic field are distributed in a large range of directions.

To calculate the power spectrum we use the fast Fourier transform (FFT). Aiming to remove the least well-determined elements of the spectrum and eliminate noise from our results, we discarded the first 5 points and kept only the subsequent 50 points for each spectrum. The corresponding frequency range is $7.8 \times 10^{-3} \mathrm{~Hz}$ to $7.16 \times 10^{-2} \mathrm{~Hz}$. In Fig. 2 we show an example of the power spectrum for one of the arrays.

\section{Results}

A power law was fitted to the spectra. The values of the spectral index $(q)$ that result from our calculations are generally smaller than the Kolmogoroff value $(-5 / 3)$. The median of the spectral index for the 284 spectra is $-1.07 \pm 0.37$. There is no statistically significant differ- 


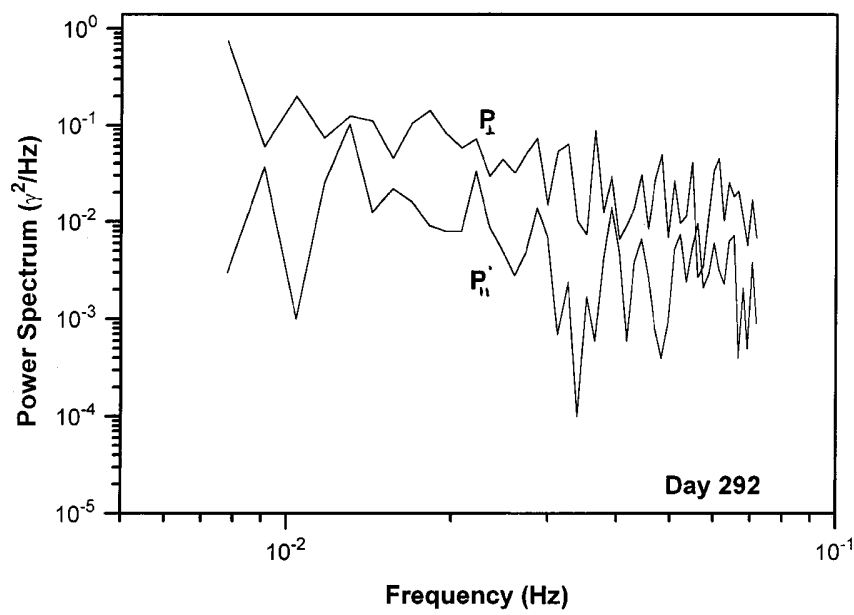

Fig. 2. An example of power spectra perpendicular and quasi-parallel to the mean field inside the CIR

ence between the values of $q$ for quasi-parallel or perpendicular spectra.

To obtain the ratio of perpendicular $\left(P_{\perp}\right)$ to quasiparallel $\left(P_{\|}^{\prime}\right)$ spectra we use the intermediate frequency of our interval $\left(3.97 \times 10^{-2} \mathrm{~Hz}\right)$.

In Table 1 we present the median value of the spectral index $q$ and the weighted average of $P_{\perp} / P_{\|}^{\prime}$. All the values are smaller than 1 . This means that, on average, the parallel power is greater than the perpendicular power. This is contrary to the results of Bieber et al. (1996) where this ratio is always greater than unity.

Finally we calculated the ratio $P_{\perp} / P_{\|}^{\prime}$ and the standard deviation for each class interval defined every $10^{\circ}$. The outcome of this analysis is presented in Figs. 3-5. In each figure the value of the ratio for each class, is positioned at the average angle for that class. In all three figures the errors represent the dispersion around the average value.

Due to poor statistics it is difficult to make an attempt to fit any kind of curve to our results. The best fit possible is a horizontal line at the average value before the forward shock. This indicates that the fluctuations have more power in the direction quasiparallel to the radial.

Inside the CIR the mean value of the ratio $(0.74 \pm 0.43)$ is not inconsistent with a value of 1 , which would indicate slab type fluctuations. Note that in this region the average field bends away from the radial direction.

Table 1. Mean values of the spectral index and the ratios of the spectra before, during and after the CIR

\begin{tabular}{lccc}
\hline & Before the CIR & In the CIR & After the CIR \\
\hline$\langle q\rangle$ & $-1.13 \pm 0.39$ & $-1.10 \pm 0.39$ & $-1.02 \pm 0.33$ \\
$\begin{array}{l}\text { (median) } \\
P_{\perp} / P_{\|}^{\prime} \\
\text { (weighted average) }\end{array}$ & $0.66 \pm 0.30$ & $0.74 \pm 0.43$ & $0.64 \pm 0.37$ \\
\hline
\end{tabular}

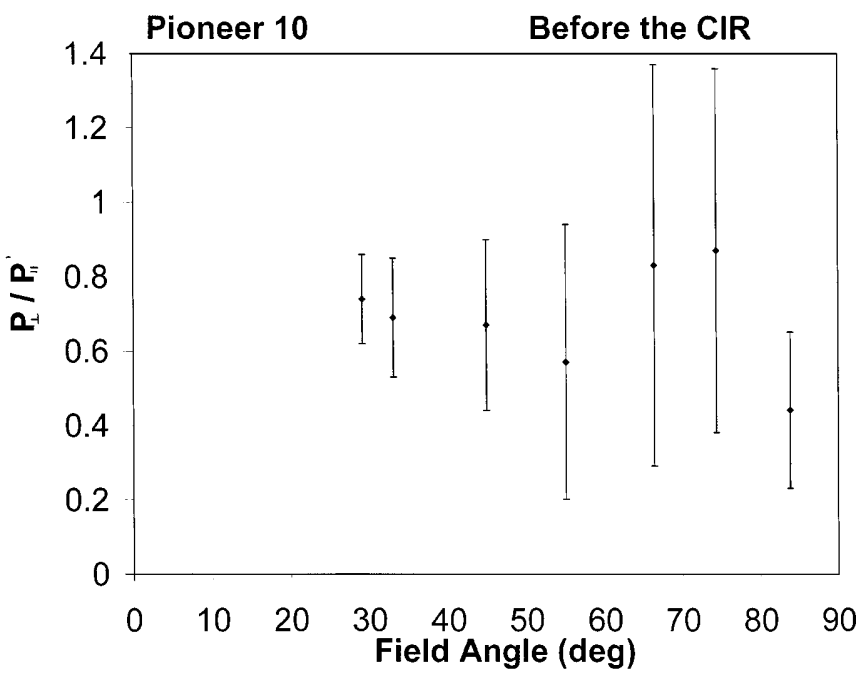

Fig. 3. Ratio $P_{\perp} / P^{\prime}$ as a function of the angle of the mean magnetic field for the interval before the CIR

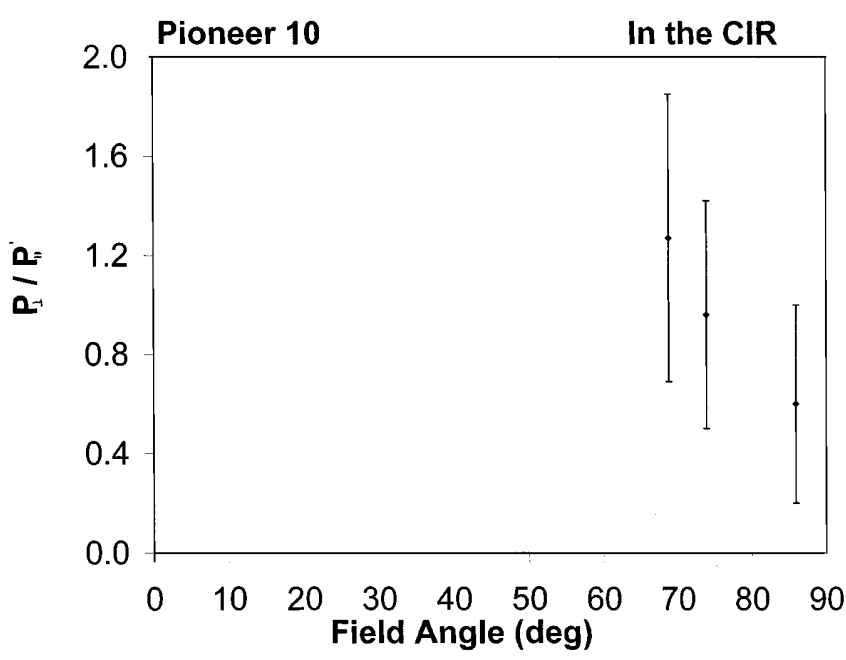

Fig. 4. Ratio $P_{\perp} / P_{\|}^{\prime}$ as a function of the angle of the mean magnetic field within the CIR

After the CIR reverse shock, fluctuations are again much more important in the quasi-parallel direction than in the perpendicular direction.

The interpretation of the symmetry of the fluctuations depends critically on a well-defined average magnetic field direction. The test of ergodicity as represented by the autocorrelation function being a Lanczos type function guarantees that the time average of the IMF data samples over an interval $T\left([B]_{T}\right)$ converge to the ensemble average $b$, where $b$ is independent of time (Matthaeus and Goldstein, 1982b). Nevertheless, we went beyond the ergodicity test and a considerable more restrictive condition was imposed on the data samples before and after the CIR, where fluctuations are clearly larger in the quasi-parallel direction than in the perpendicular direction. The condition corresponds to fluctuations that are completely uncorrelated for times $t>T_{0}$, where the autocorrelation function is $R\left(t>T_{0}\right) \approx 0$, and may be expressed as: 


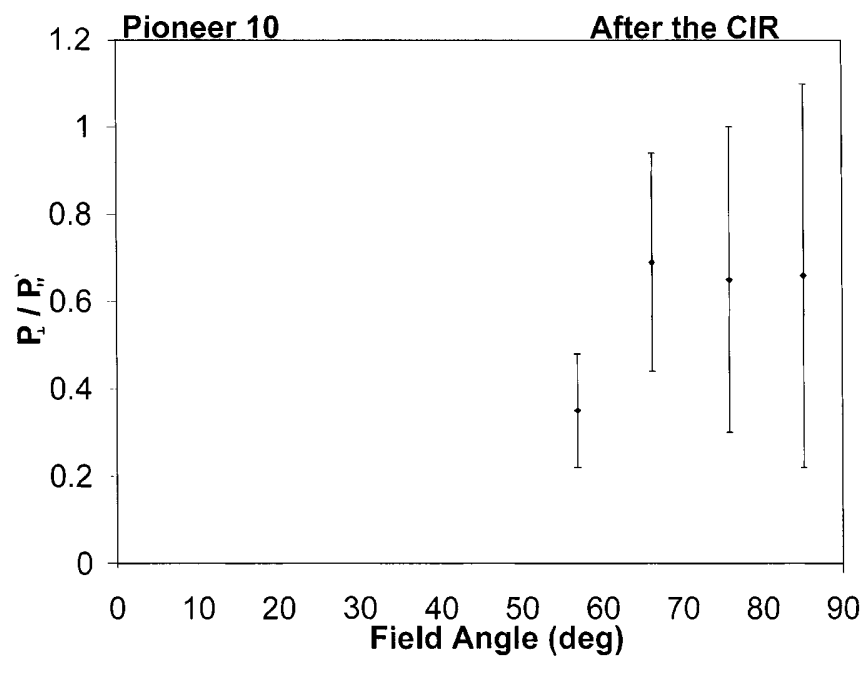

Fig. 5. Ratio $P_{\perp} / P_{\|}^{\prime}$ as a function of the angle of the mean magnetic field for the interval after the CIR

$\Delta^{2}[B]_{T} \equiv\left\langle\left([B]_{T}-b\right)^{2}\right\rangle=2 \sigma^{2} T_{c} / T+O\left(T_{c} / T\right)^{2}$

(Matthaeus and Goldstein, 1982b).

In Fig. 6 we show plots of $\Delta^{2}[B]_{T}$ normalised by $\sigma^{2}$ versus $T_{c i} / T$, where $T_{c i}$ is the corresponding correlation time for $i=x, y, z$. The values of $\Delta^{2}[B]_{T}$ and $\sigma^{2}$ were calculated following Matthaeus and Goldstein (1982b). The plots correspond to data taken on day 292 , in the region "before the CIR". A similar behaviour is observed when calculations are made with data "after the CIR". All three components show a fast convergence as $T$ exceeds $T_{c}$, with the $z$ component converging faster than the others. Thus the second order moments apparently converge rapidly enough to guarantee that we are dealing with fluctuations that are completely uncorrelated.

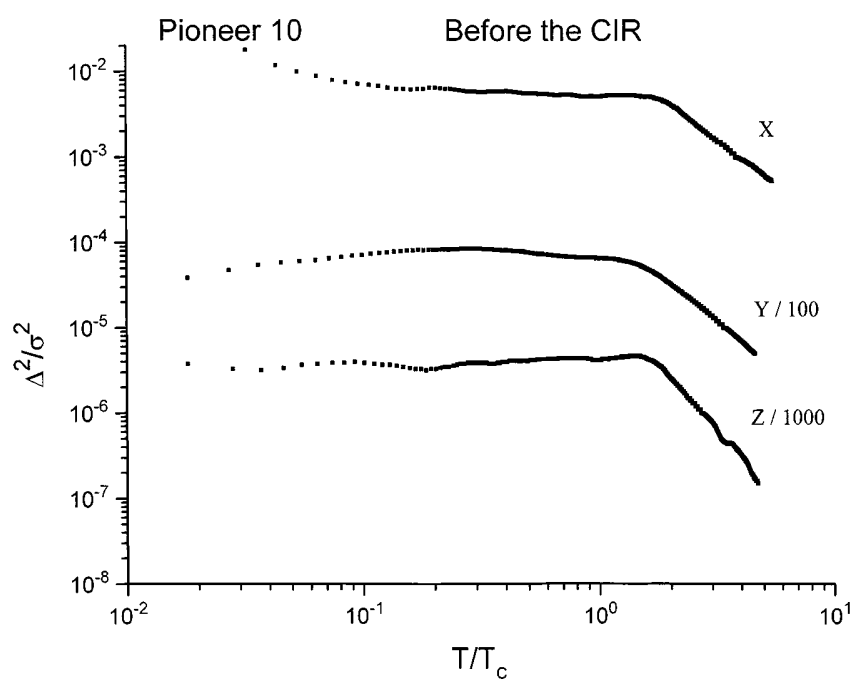

Fig. 6. The variance of the means $\Delta^{2}[B]_{T}$ normalised by $\sigma^{2}$ for the $x$, $y$ and $z$ field components of data taken on day 292, "before the CIR", calculated for intervals $T$ and plotted as functions of $T_{c i}$, the corresponding correlation time
Having established the fast convergence of the averages we may now summarise our results, with the Bieber et al. (1996) model as reference, to say that before and after the CIR fluctuations are predominantly quasi-parallel and within the CIR fluctuations could be slab-like. An interpretation of the geometry of the fluctuations in terms of the composite slab/2D model of Bieber et al. (1996) is not possible in our case. Therefore we offer plausible alternative explanations in the following section.

\section{Discussion}

In this work we perform a study of the geometry of the magnetic fluctuations before the forward shock, inside and after the reverse shock of a corotating interaction region observed by Pioneer 10 at 5 AU during days 292296 in 1973. To do this we calculate the spectra perpendicular and parallel to the mean magnetic field according to the methodology proposed by Bieber et al. (1996). These authors designed a composite slab/2D model for the magnetic fluctuations and applied it to the data obtained by the Helios spacecrafts between 0.3 and 1 AU. In that work, they estimated that approximately $85 \%$ of the energy of the fluctuations is in the $2 \mathrm{D}$ component and concluded that this component is predominant over the slab component. The average value of the ratio of the perpendicular and the parallel spectra to the mean magnetic field obtained by Bieber et al. (1996) was 1.4, larger than 1, that corresponds to the slab case, and smaller than 1.67 as it would be in the pure 2D model. We obtained appreciable differences with respect to the results of these authors. It is evident that the composite model proposed by Bieber et al. (1996) can not be applied to our results.

We expect that near the forward and reverse shocks the physical processes of compression and rarefaction of the plasma play an important role to generate the magnetic field fluctuations. For that reason compressive fluctuations should be considered in the analysis of the fluctuating field. In particular, we may consider the presence of the magnetoacoustic mode. Moreover, the main assumptions of the composite model may not be necessarily applied in the conditions of the CIR where strong interactions of the MHD fluid between the fast and slow streams are at work. In this sense it is possible that the condition of axial asymmetry with respect to the mean magnetic field may not be valid, and that the fluctuations may be aligned to another direction, which could be the radial or a direction associated with the shock wave front. Therefore it is not surprising that the model in question did not work under these particular circumstances. Nevertheless the calculations served as a confirmation of one of the limitations of the Bieber et al. (1996) model and the need to consider alternatives to describe the IMF turbulence around CIRs.

The composite model proposed by Bieber et al. (1996) enforced additional condition that the power of the fluctuations along the mean magnetic field $\left(P_{z z}\right)$ were 
negligible with respect to the perpendicular fluctuations. That condition was justified based on the results of Belcher and Davis (1971). This is not strictly true for the data analysed here, in many cases the power spectrum parallel to the direction of the mean magnetic field was of the order of the perpendicular power spectrum.

Let us first discuss the results of the region within the CIR. From Table 1 we see that this is the only case where the value of the ratio is statistically consistent with 1 , at a first view that suggests predominance of the slab geometry. Nevertheless, analysing the values of the angles of the mean IMF (Fig. 3) one realises that these are larger than $60^{\circ}$, that is, the mean field is almost perpendicular to the radial direction. For these angles the composite slab/2D model of Bieber et al. (1996) presents the major deviations with respect to their own experimental results (see their Fig. 1). This is an indication that perhaps we should look for an alternative model to explain our findings.

If we note that the quasi-parallel spectrum can be decomposed in a component parallel to the radial direction and another perpendicular to it, and if we take into account the fact that the fluctuations parallel to the direction of the mean magnetic field may not be neglected and that for angles $\Psi$ close to $90^{\circ}$ this component contributes mainly to the spectrum perpendicular to the radial direction, then the ratio $P_{\perp} / P_{\|}^{\prime}$ will increase considerably. A plausible interpretation of this may be that we are in the presence of Alfvenic fluctuations propagating in the radial direction. This is in agreement with the results of Mavromichalaki et al. (1988). Making a different analysis of the same CIR, these authors found evidence of the presence of large amplitude Alfvén waves propagating near to the radial direction. Hence, we reject the interpretation of a slab geometry of the fluctuations within the CIR. The situation is in fact much closer to a $2 \mathrm{D}$ structure of the fluctuations since the propagation direction (radial) is nearly perpendicular to the IMF. The model is not able to accommodate our results due to the explicit neglect of the fluctuations parallel to the average IMF. On the other hand, an inclusion of $P_{z z}$ would make it impossible to have a simple analytical expression for the ratio $P_{\perp} / P_{\|}^{\prime}$, with which we could make comparisons. Nevertheless it is remarkable that the results of our analysis admit an interpretation in terms of 2D turbulence. This might be an indication that the turbulence structure was preserved inside the CIR from the inner heliosphere out to $5 \mathrm{AU}$.

How can we interpret the outcome of our work outside the CIR? The theoretical model of Bieber et al. (1996) neither work here since the averages of $P_{\perp} / P_{\|}^{\prime}$ are always less than one. Therefore we should look for a different theoretical context for our results.

Kunstmann (1978) made an analysis of four models that are employed in the study of IMF fluctuations: (a) slab model (fluctuations propagating parallel to the mean field), (b) axisymmetric Alfvén waves (equivalent to a composite slab 2D model), (c) isotropic waves, and (d) magnetoacoustic waves. He was able to deduce analytical forms of the spectral matrix in each of the four cases. For his study Kunstmann (1978) considered a system of coordinates where the parallel direction is the direction of mean magnetic field and points towards the Sun, the direction " $E$ " is in the ecliptic plane and points toward positive $y$ values in the solar ecliptic system, and the direction " $N$ " completes the right handed system. Likewise, the spectrum parallel to the mean magnetic field is $P_{\|}^{K}$, and the spectrum perpendicular to the direction of the mean magnetic field by $P_{\perp}^{K}=\frac{1}{2}\left(P_{E E}+P_{N N}\right)$, where $P_{E E}$ and $P_{N N}$ are the power spectra of directions " $E$ " and " $N$ " respectively.

In this system Kunstmann (1978) arrives at an expression for the ratio of spectra $P_{\|}^{K} / P_{\perp}^{K}$ as a function of the spiral angle when the mean magnetic field is in the ecliptic plane $\left(\theta=0^{\circ}\right)$ and obtains the behaviour of this ratio for different combinations of the four mentioned models.

In order to realign our spectra to the Kunstmann (1978) theoretical models we had to rotate our IMF data accordingly and calculate the new spectra and ratios $P_{\|}^{K} / P_{\perp}^{K}$. During this process we observed that the average zenith angle of our samples was not zero, as required by the Kunstmann (1978) analysis. Nevertheless our data did not show average field directions larger than $20^{\circ}$ and the attempt to interpret our spectral ratios in terms of the Kunstmann (1978) models will have some meaning at least qualitatively.

Figure 7 shows our results for the region before the CIR. This result can be interpreted as a combination of the isotropic and the magnetoacoustic models. According to the theoretical model of Kunstmann (1978), the ratio $P_{\|}^{K} / P_{\perp}^{K}$ for magnetoacoustic waves has a behaviour similar to that in Fig. 7, i.e., the ratio decreases with increasing spiral angle of the mean magnetic field if the spectral index is close to one, as is our case. However the ratio obtained here ranges from values around unity to nearly 0.2 . These values of the ratio are more typical of isotropic fluctuations according to Kunstmann (1978). Qualitatively we may therefore say that before the CIR,

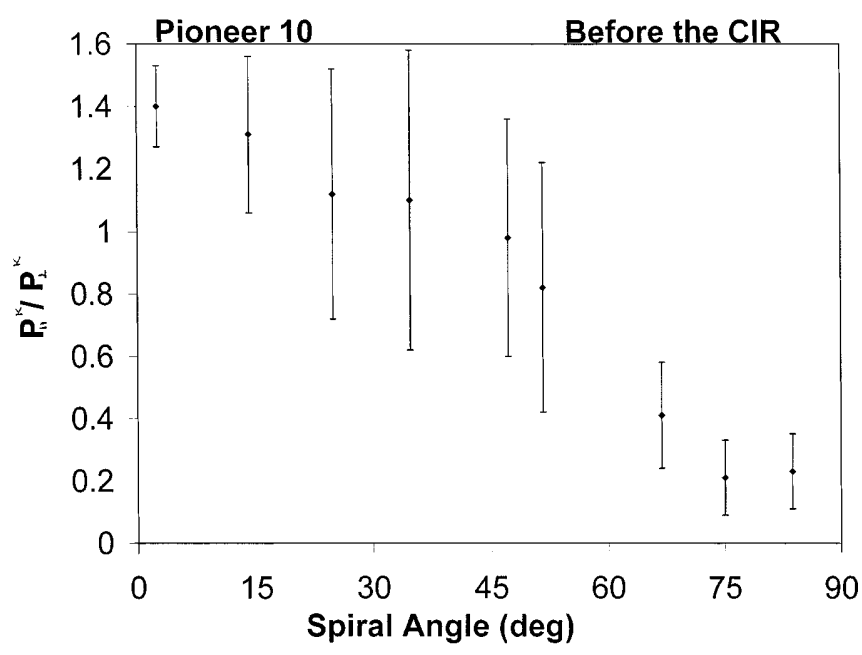

Fig. 7. Ratio of parallel to perpendicular spectra as a function of the spiral angle for the interval of time before the CIR. Directions of spectral components are defined according to Kunstmann (1978) 
the IMF have fundamentally two components: one isotropic and another magnetoacoustic.

If we now make the same calculation for the time interval after the CIR, we obtain the plot that appears in Fig. 8. According to the work of Kunstmann (1978), this ratio could be representative of a combination of the axisymmetric Alfvén waves (composite slab-2D) with a proportion of around $30 \%$ of magnetoacoustic waves (see his Fig. 4).

We emphasise that the results, shown in the last two figures, are only qualitative. Better statistics and a sample where the vectors of the average magnetic field lie closer to the ecliptic plane would be necessary to make a quantitative comparison of experimental results with theory. Nevertheless it is comforting that at least a qualitative comparison can be made with a theoretical model. Moreover our results here serve to call attention to the importance that magnetoacoustic fluctuations may have in some regions of the interplanetary medium.

It may seem unusual that we have unified the data inside the CIR for our analysis since there two different plasmas exist, separated by an interface. However we did first analyse the spectra separately and did not find any significant difference between them. On the other hand the criteria of stationarity applied to extract the useful data forced us to discard a good proportion of the original measurements, therefore separating the data within each of the regions of the CIR would result in very poor statistical inputs from which no conclusions could be extracted.

Finally we would like to stress that the model proposed by Bieber et al. (1996) was applied to a set of data representative of the region between 0.3 to $1 \mathrm{AU}$ from the Sun. There the stream interaction has not yet taken place as opposed to the $5 \mathrm{AU}$ region where a full CIR has had enough time to develop completely. In that sense it is remarkable that fluctuations inside the CIR, far from the compression regions are found to be of Alfvenic nature and in qualitative agreement with the

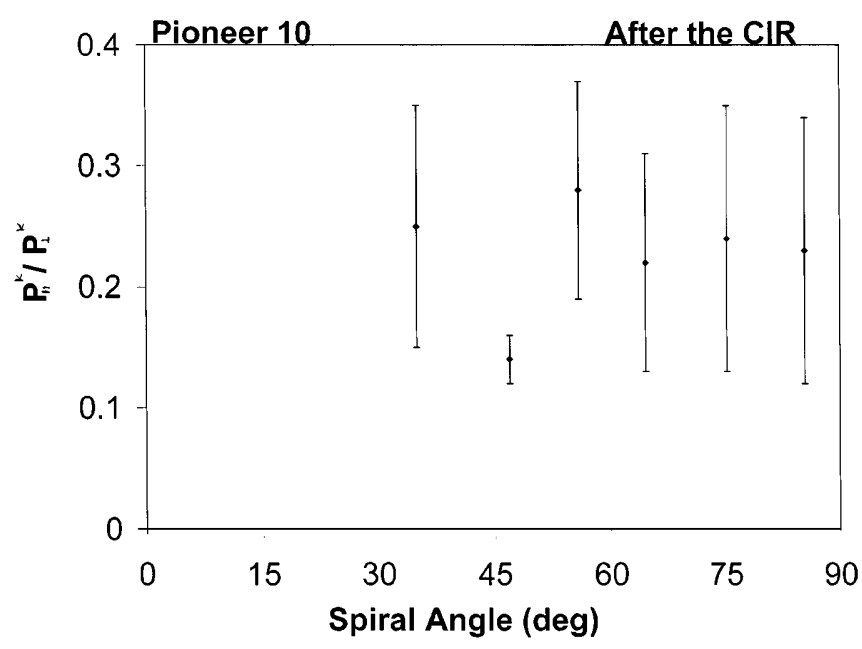

Fig. 8. Ratio of parallel and perpendicular spectra as a function of the spiral angle for the interval of time after the CIR, according to Kunstmann (1978) predicitions of a 2D dominated model. The results outside the CIR came as no surprise since these regions do not fulfill some of the model assumptions. On the other hand we were able to find a qualitative interpretation of our results in the latter regions considering a significant proportion of magnetoacoustic waves in the observed fluctuations.

\section{Conclusions}

In this work we performed a study of the geometry of the IMF fluctuations in a corotating interaction region observed by Pioneer 10 at 5 AU on days 292-296 in 1973. We tried to apply the composite slab/2D model proposed by Bieber et al. (1996), that has successfully explained the turbulence geometry of a large set of data in the inner heliosphere (see also Leamon et al., 1998). We proved that the magnetic fluctuations in this CIR are not in agreement with the predictions of this model. We found a qualitative explanation of our results before the forward shock and after the reverse shock based in the theoretical analysis of Kunstmann (1978) for the regions outside the CIR. Inside the CIR our results and previous work done by Mavromichalaki et al. (1988) indicate a predominance of a 2D geometrical turbulence which would fit in a less restrictive model.

Our conclusions are the following:

1. The composite slab/2D model proposed by Bieber et al. (1996) is not completely applicable to the regions near or within our CIR.

2. In the region before the studied CIR (next to the forward shock), the magnetic fluctuations are a combination of isotropic and magnetoacoustic waves, as could be appreciated when comparing our results with those of the theoretical study made by Kunstmann (1978). This theoretical model predicts, that for this combination of the fluctuations, the ratio of the parallel to the perpendicular spectrum with respect to the mean magnetic field decreases with the increase of the average IMF spiral angle as our results indicate (see Fig. 7).

3 . In the region within the analysed CIR, the fluctuations are Alfvenic, propagating near to the radial direction and with vectors of the mean magnetic field almost perpendicular to the radial direction. Here the disagreement with Bieber et al. (1996) is mainly due to the restriction imposed in their model where they neglect the power contained in fluctuations of the component parallel to the average IMF. Nevertheless, our results are in accordance with a geometry where there is a dominant 2D component in the turbulence. This conclusion is in accordance with one of the results of the study performed to this CIR by Mavromichalaki et al. (1988); they arrived at the conclusion that within this CIR the fluctuations are Alfvenic, with nearly radial wave vectors.

4. According to the results of Kunstmann (1978) in the region after the CIR, the fluctuations have a behaviour in agreement with a mixture of Alfvén waves with axial symmetry and magnetoacoustic waves. 
Acknowledgements. One of us (RC) wishes to express gratitude to the Mexican Ministry of Foreign Affairs for a scholarship during the course of this research. This work was partially supported by CONACyT grant L0047T.

Topical Editor R. Schwenn thanks M. L. Goldstein and another referee for their help in evaluating this paper.

\section{References}

Belcher, J. W., and L. Davis, Jr., Large-amplitude Alfvén waves in the interplanetary medium, J. Geophys. Res., 76, 3534, 1971.

Bieber, J. W., W. H. Matthaeus, C. W. Smith, W. Wanner, M.-B. Kallenrode, and G. Wibberenz, Proton and electron mean free paths: the Palmer consensus revisited, Astrophys. J., 420, 294 306, 1994.

Bieber, J. W., W. Wanner, and W. H. Matthaeus, Dominant twodimensional solar wind turbulence with implications for cosmic ray transport, J. Geophys. Res., 101, 2511-2522, 1996.

Blackman, R. B., and J. W. Tukey, The measurement of power Spectra, Dover, New York, 1958.

Hasselmann, K., and G. Wibberenz, Scattering of charged particles by random electromagnetic fields, Z. Geophys., 34, 353, 1968.

Jokipii, J. R., Cosmic ray propagation. I. Charged particles in a random magnetic field, Astrophys. J., 146, 480, 1966.

Kolmogoroff, A. N., The local structure of turbulence in incompressible viscous fluid for very large Reynolds numbers, $C . R$. Acad. Sci. URSS, 30, 201, 1941.

Kunstmann, J. E., The dependence of the frequency spectra of the interplanetary magnetic-field fluctuations on the direction of the mean field, The Moon and the Planets, 18, 91-104, 1978.

Kunow, H., G. Wibberenz, G. Green, R. Muller-Mellin, and M.-B. Kallenrode, Energetic particles in the inner solar system, in
Physics of the inner-heliosphere, Eds R. Schwenn and E. Marsh, Springer, Berlin Heidelberg New York, pp 243/342, 1991.

Leamon, R. J., C. W. Smith, N. F. Ness, W. Matthaeus and H. K. Wong, Observational constraints on the dynamics of the interplanetary magnetic field dissipation range, J. Geophys. Res., 103, 4775-4787, 1998.

Matthaeus, W. H., and M. L. Goldstein, Measurements of the rugged invariants of magnetohydrodynamic turbulence in the solar wind, J. Geophys. Res., 87, 6011-6028, 1982a.

Matthaeus, W. H., and M. L. Goldstein, Stationarity of magnetohydrodynamic fluctuations in the solar wind, 87, 10 347-10 354, $1982 b$.

Matthaeus, W. H., M. L. Goldstein, and D. A. Roberts, Evidence for the presence of quasi-two-dimensional nearly incompressible fluctuations in the solar wind, J. Geophys. Res., 95, 20 673, 1990.

Matthaeus, W. H., J. W. Bieber, and G. P. Zank, Unquiet on any front: anisotropic turbulence in the solar wind, Rev. Geophys. Suppl., 609, 1995.

Mavromichalaki, H., X. Moussas, J. J. Quenby, J. F. ValdésGalicia, E. J. Smith, and T. Thomas, Relatively stable, largeamplitude alfvénic waves seen at 2.5 and 5 AU, Solar Phys., 116, 377-390, 1988.

Oughton, S., E. Priest, and W. Matthaeus, The influence of a mean magnetic field on 3D MHD turbulence, J. Fluid Mech., 280, 95, 1994.

Robinson, D., and M. Rusbridge, Structure of turbulence in the zeta plasma, Phys. Fluids, 14, 2499, 1971.

Valdés-Galicia, J. F., Energetic particle transport coefficients in the heliosphere, Space Sci. Rev., 62, 67-93, 1993.

Zweben, S., C. Menyuk, and R. Taylor, Small-scale magnetic fluctuations inside the Macrotor tokamak, Phys. Rev. Lett., 42, 1270, 1979. 